\title{
High resolution, high throughput Electron Tomography reconstruction
}

\author{
R.H.M. Schoenmakers, R.A. Perquin, T.F. Fliervoet, and W. Voorhout \\ FEI Company, Achtseweg Noord 5, 5600 KK, Eindhoven, The Netherlands
}

To obtain high quality 3D sample data by using electron tomography, there are several aspects of major importance, both on the TEM during the acquisition of data as well as in the subsequent reconstruction process. The acquisition on the Tecnai $\mathrm{G}^{2} \mathrm{TEM}$ microscope is optimized by means of the concepts of (holder) calibration and optimized position and by using an internally consistent set of alignments and calibrations. This paper will show that the only way to achieve high resolution with high throughput (high speed) tomography is to have the processes of reconstruction of the data closely linked to that of acquisition, as is done in our approach using Xplore3D. The fewer image artifacts introduced by optimizing the acquisition, the more accurate and faster the subsequent image alignment and 3D reconstruction can be [1].

High resolution details in the final reconstructed tomogram can only be achieved using very accurate alignment of the tilt series images. Both the process of high accuracy cross correlation alignment and that of both manual and automated feature and marker tracking will be described. Differences between the results of these alignment techniques will be shown and application areas of each technique are discussed. An example is given in Figure 1.

The classical reconstruction technique of weighted back-projection (WBP) is limited by both noise and the loss of both high and low frequency information (Figure 2). These effects are better dealt with by iterative techniques such as ART [2] and SIRT [3]; both are incorporated in $\operatorname{Inspect3D^{(*)}}$. These techniques do not rely on filtering, but rather iteratively optimize the tomograms so projections of the reconstructions best resemble the acquired data. In this case, all spatial frequency information from the original data is used to produce the final tomogram. The superior quality of SIRT reconstructions is shown using examples from Life Science, Material Science and the semiconductor industry (an example is given in Figure 2).

Typically, these iterative reconstruction techniques are slow: every iteration consists of a projection and a back-projection, which take about the same amount of time. So a 15 iteration SIRT reconstruction will take about 30 times longer than a single WBP reconstruction, which in the case of large, multiple Gigabyte data sets becomes prohibitively long (days rather than hours or minutes). We will present a way of accelerating these reconstruction techniques on a single desktop PC without the need for any dedicated hardware and show some preliminary results. The estimated speedup is a factor of 20 , bringing the iterative techniques back into the scope of practical work, and accelerating the weighted back projection to almost interactive speeds.

\section{References}

[1] Xplore3D white paper, FEI Company.

[2] R. Gordon, R. Bender, G.T. Herman, J.Theor.Biol. 29 (1970) 471.

[3] P. Gilbert, J.Theor.Biol. 36 (1972) 105. 
(*) We gratefully acknowledge Dr. M. Weyland, Cambridge University, UK, for cooperation on the implementation of these algorithms.
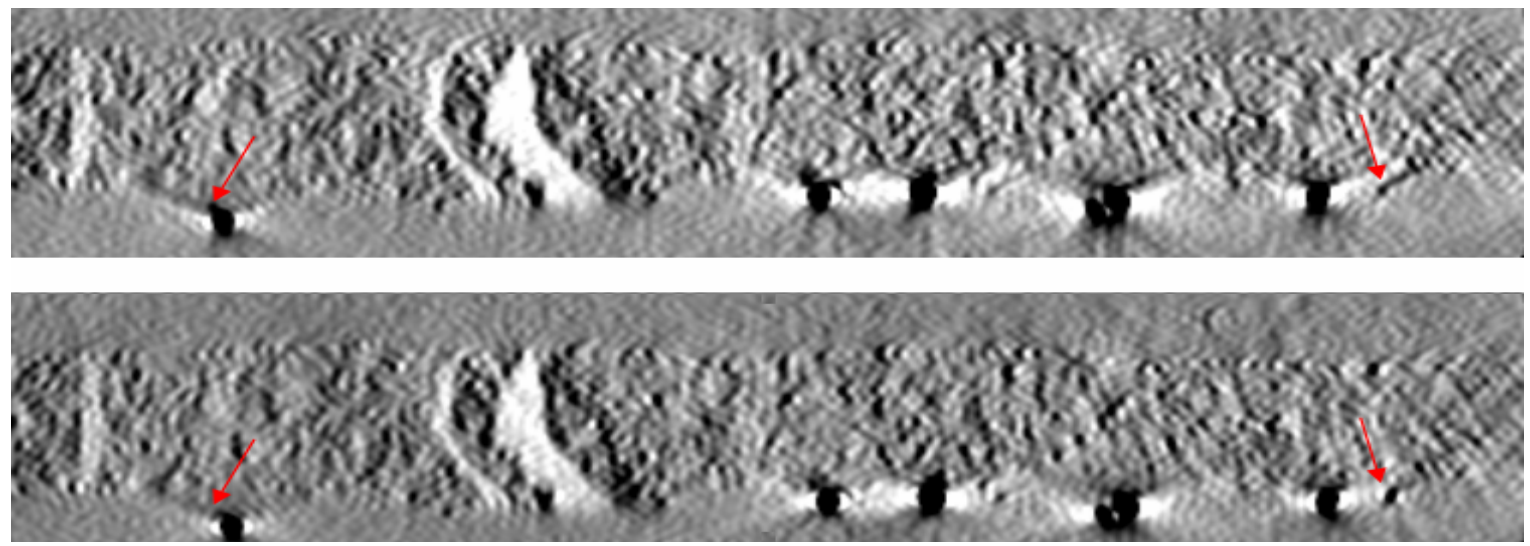

Fig. 1. Comparison between WBP reconstructions calculated using a purely cross correlation based alignment (top) and using alignment by feature tracking (bottom). The feature tracking also corrects for magnification changes and rotation of the position of the tilt axis in each image of the tilt series. The resulting image shows that feature tracking produces more accurate results (see arrows). It should be noted that for many specimens that do not contain markers the cross correlation still gives accurate results.
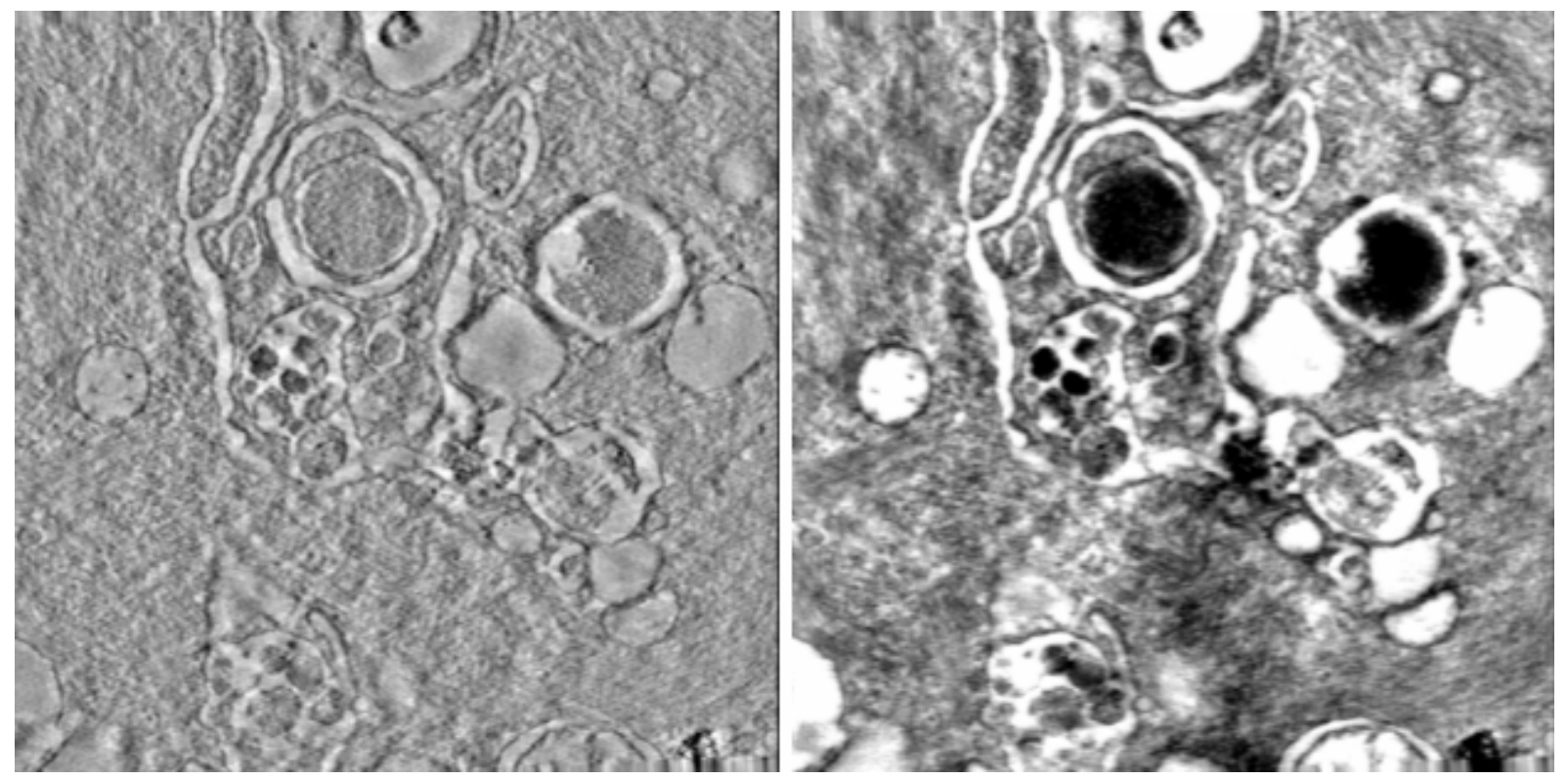

Fig. 2. Comparison between (left) a WBP reconstruction and (right) a SIRT reconstruction of 15 iterations of vesicle-like structures in notochordal cells from the nucleus pulposi of canine lumbar spines. Both pictures display the same slice from the reconstructed volume. The data set was aligned by cross correlation only (no gold markers were applied to the set). The increased information content in the SIRT reconstruction is striking. Data kindly provided by Christopher J Hunter, Jonathan Chung \& Matthias Amrein, University of Calgary (Canada). 